\title{
Therapeutic targeting of tumor cells rich in LGR stem cell receptors
}

Songman $\mathrm{Yu}^{\#}$, Maria Carmen Mulero\#, Wannan Chen, Xiying Shang, Songyu Tian, Joji Watanabe, Arisa Watanabe, Tim Vorberg, Clara Wong, Dennis Gately and Stephen B. Howell*.

Songman Yu, MD, Department of Infectious Diseases, Hunan Key Laboratory of Viral Hepatitis, Xiangya Hospital, Central South University, Changsha, 410008, China

Maria Carmen Mulero, PhD, UCSD Moores Cancer Center and Department of Medicine, University of California, San Diego, 3855 Health Sciences Drive, Mail Code 0819, La Jolla, CA 92093-0819, USA

Wannan Chen, PhD, Key Laboratory of Gastrointestinal Cancer, Ministry of Education, School of Basic Medical Sciences, Fujian Medical University, 1 Xue Fu North Road, Fuzhou, Fujian 350122, China

Xiying Shang, MD, PhD, UCSD Moores Cancer Center and Department of Medicine, University of California, San Diego, 3855 Health Sciences Drive, Mail Code 0819, La Jolla, CA 92093-0819, USA

Songyu Tian, MD, Department of Gynecology, Harbin Medical University Cancer Hospital, Harbin, Heilongjiang Province, 150081, China

Joji Watanbe, MD, Department of Surgery, Division of Surgical Oncology, Tottori University Hospital, 36-1 Nishi-cho, Yonago, 683-8504, Japan

Arisa Watanabe, MD, Akasaki Medical Office, 1920-74 Akasaki, Kotoura-town, Tohakudistrict, Tottori-prefecture, 689-2501, Japan

Tim Vorberg, Heidelberg Institute for Stem Cell Technology and Experimental Medicine, Division of Cancer Stem Cells and Metastases, Im Neuenheimer Feld 280, 69120 Heidelberg, Germany

Clara Wong, UCSD Moores Cancer Center and Department of Medicine, University of California, San Diego, 3855 Health Sciences Drive, Mail Code 0819, La Jolla, CA 92093-0819, USA

Dennis Gately, PhD, UCSD Moores Cancer Center and Department of Medicine, University of California, San Diego, 3855 Health Sciences Drive, Mail Code 0819, La Jolla, CA 92093-0819, USA

\section{Corresponding Author}

**To whom correspondence should be addressed: Stephen B. Howell, MD, Moores UCSD Cancer Center, 3855 Health Sciences Drive, Mail Code 0819, La Jolla, CA 92093-0819, USA;

Tel. 1-(858) 822-1110; Fax 1-(858) 822-1111; email: showell@health.ucsd.edu 


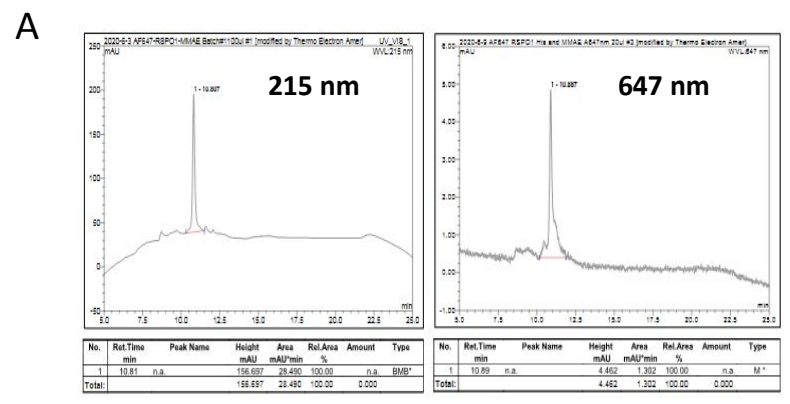

B

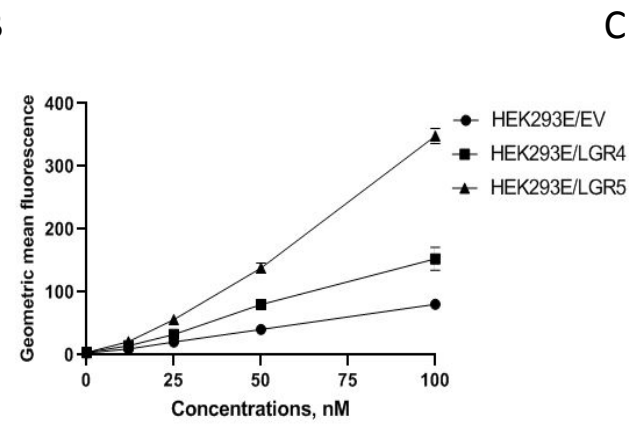

C

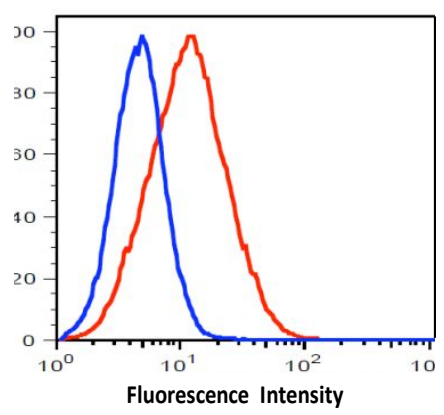

Figure S1. A, HPLC analysis of AF647-R1FF-MMAE at 215 and $647 \mathrm{~nm}$ documenting successful AF647-labeling. B, Differential binding of AF647-R1FF-His to HEK293/EV, HEK293/LGR4 and HEK293/LGR5 cells as a function of concentration. C, Flow cytometric analysis of LGR5 expression in HEK293/EV (blue) and HEK293/LGR5 (red) cells; the ratio of the mean fluorescent intensities is 26.

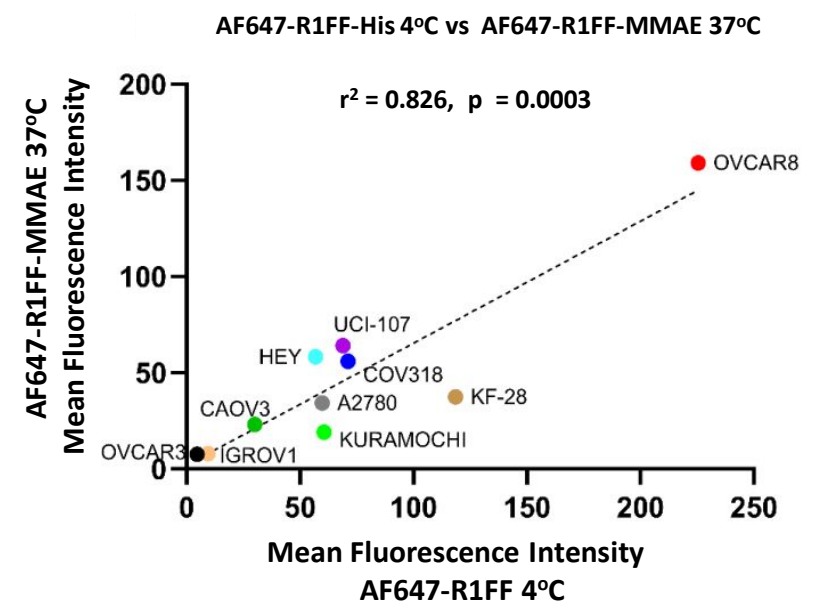

Figure S2. Correlation between the binding of AF647-R1FF-His at $4^{\circ} \mathrm{C}$ and the uptake of AF647-R1FF-MMAE at $37^{\circ} \mathrm{C}$ in a panel of 10 human ovarian cancer cell lines. 